\title{
Study on optimal path of intelligent rural tourism Based on the integrated navigation grid model
}

\author{
Tong Wu ${ }^{1,}$ a Min Huan ${ }^{2}$ \\ ${ }^{1}$ Department of Tourism and Foreign Languages, Ma'anshan Teachers' College, Ma'anshan \\ 243041, China \\ ${ }^{2}$ Department of Applied Foreign Languages, Ma'anshan Technical College, Ma'anshan 243041, \\ China \\ awtwarner@126.com
}

Keywords: Optimal path, Intelligent rural tourism, Integrated navigation grid model.

Abstract: Path planning technology plays an important role in travel path optimization. In this paper, the wisdom of rural tourism scenic spots laying ropeway abstract graph for the shortest path model, using the minimum spanning tree for expression. Floyd algorithm-cluster analysis method is used to establish and solve the model, and it can get the optimal solution of the problem. In view of the above advantages and disadvantages of the Floyd algorithm-cluster analysis model, this article gives specific improvements to make it more realistic and save the most money.

\section{Introduction}

Rural tourism is a form of village tourism, which is based on the principle of traveling and vacation; we can take the outdoor field in the village as a place, or take the humanities as non-interference, ecological and non-destructive, and featuring touristy[1,2]. With the rapid development of rural tourism, many original and new concepts and theories have put forward around rural tourism in recent years[3]. The introduction of concepts and new theories enriches rural tourism and diversifies its forms, effectively alleviating the growing problem of homogenization of rural tourism.

In the past, rural tourism went to the villages to understand some rural customs, customs and ceremonies, as well as some local products, fruit trees, brooks, bridges and their stories that have planted. Visitors can stay, study, experience rural lifestyle activities in and around the village, which is often the traditional village in remote areas[4]. The village also serves as a base for travelers to explore the nearby area. The concept of rural tourism contains two aspects, which are occurred in rural areas, and rural tourism as a tourist attraction, both indispensable[5,6].

Vigorously developing rural tourism is an important part of building a new socialist countryside. It is an important way to speed up the coordinated development of urban and rural economy, realize industrial linkage, and bring the city to the countryside. It is of great significance to accelerating the development of new rural areas, coordinating the urban and rural development, and increasing the employment opportunities for peasants[7,8]. To meet tourist demand for tourist culture and other aspects are of great significance. 
Over the years, Guangzhou Wisdom actively integrated into the development of Chinese rural tourism. It has successively served as a village healthy tourism project under the health and retirement healthy village in Wenzhou under the Dongshan Mountain, the most beautiful village in the Maotian Pingjiang of Hunan Province, and the beautiful rural carnival in Chongqing, which enriched the prosperity of rural tourism development. Among them, the grasping of the two major advantages of rural tourism in the recreation and recreation build Wenzhou Dongshan under the health and retirement health village is a collection of local culture and leisure health typical cases, the culture of living, the landscape needs to be spiritual.

\section{Problems in rural tourism}

With the continuous improvement of people's lives, tourism has become an important activity to improve people's quality of life. However, time is often a problem that limits people's travel, in order to meet the needs of tourists. The tourism industry is now more developed, but many people hope to find the best tourist routes because of the time constraints. The most direct problem encountered by the tourist routes are the specific location of attractions.

For example, Zhangjiajie scenic spots, as many as fifty spots, it has become a key issue to be solved. To this end, Zhangjiajie scenic decided to lay the cable car cableway to solve the problem of the majority of tourists'time. For Zhangjiajie scenic spots to do the standard problem, first locate the attractions in the map of the latitude and longitude; and then use computer technology to deal with longitude and latitude, and then to Zhangjiajie National Forest Park as the origin of the new coordinate system to the east and north. The direction is the $\mathrm{X}$ and Y-axes of the new coordinate system, and the new Zhangjiajie plane coordinate system has created.

Finally, the specific coordinates of each attraction in the new coordinate system are given. In this paper, we first consider the ideal situation of the air cable car stations built by various scenic spots in Zhangjiajie, that is, build a cable car tourist platform for tourists to sit back and sit at 51 spots in Zhangjiajie scenic spot. Taking into account geographical factors such as thunder and lightning, the attraction is that it is not possible to consider only the shortest distance of the aerial tramway and so on.

\section{Model establishment and solution}

Model 1 is an idealized model where every attraction has an aerial cable car station. According to the requirement of the model, 51 in the zhangjiajie scenic spots have air cable car station, 51 sites into o minimum spanning tree problem, which is in a network of connected graph of empowerment, looking for minimum weight support tree. Now a given network is $G=(V, E, W)$, we set $T=\left(V, E^{\prime}\right)$ to a supporting tree $G, \quad W(T)=\sum_{e \in E} W(e)$ gives the right $T$ of representation, the smallest spanning tree $G$ of the minimum spanning tree. In model, $W\left(T^{*}\right)=\min \{W(T)\}$, it represents the shortest distance between 51 sites. Construction costs because of unit length is certain, so we require air lift of the scenic spots in the minimum total cost, namely for the scenic spots in the smallest distance minimum spanning tree, the connected all the attractions of minimum support the weight of the tree. According to the above information, the Floyd algorithm is considered and implemented by Matlab.

Let's say $D_{m}$ an $\mathrm{N}$ by $\mathrm{N}$ matrix, its (i, j) elements are ${ }^{m}{ }^{m}$. If you know the length of each line segment in the graph, you can determine the matrix $D_{0}$, and eventually you want to get the most 
short-circuited matrix $D_{N}$. Floyd algorithm is calculated from the beginning $D_{0}$, and then Floyd algorithm is calculated again. Repeat the process until it is done. The calculation is as follows (Wu et al., 2017).

(1) The most short-circuit of vertex i to vertex m, which allows only the first $m$ - 1 vertex to be 1 , 2 , and $\mathrm{m}-1$ as the intermediate vertex.

(2) The most short-circuit from $m$ to vertex $\mathrm{j}$, which allows only the first $\mathrm{m}$ - 1 vertex to be $1,2,,$, and $\mathrm{m}-1$ as the intermediate vertex.

(3) The most short circuit from vertex i to vertex $\mathrm{j}$, which only allows the first m minus 1 vertex to be $1,2,,$, , and $\mathrm{m}-1$ as the intermediate vertex.

Because there is no have negative length of the loop, so the shorter road must be the most short circuit from $\mathrm{i}$ to $\mathrm{j}$, $\mathrm{m}$ before which only allows a vertex is $1,2, \ldots, \mathrm{m}$ as a vertex in the middle.

Hence, $\quad \mathrm{d}_{\mathrm{ij}}^{\mathrm{m}}=\min \left\{d_{i m}^{m-1}+d_{m j}^{m-1}, d_{i j}^{m-1}\right\}$.

\section{The improved algorithm}

Clustering analysis can be divided into three types: direct clustering method, the shortest distance clustering method and the longest distance clustering method. According to the added constraints the search point distance of not more than $500 \mathrm{~m}$ to make appropriate improvements. We can calculate the distance between two samples $n \operatorname{dij}\{\operatorname{dij}\}$, remember $\mathrm{D}$; we can calculate that the $\mathrm{n}$ templates are the smallest rectangular area; Searching out the coordinate (i, j) and the number of points $\mathrm{k}$ represented by the most dense area where the scenic area satisfies the constraint condition. According to the above clustering algorithm steps, the optimal cableway cable can be obtained by using the Floyd algorithm in the minimum spanning tree method. Specific operations can be as follows. Due to the national forest park as the starting point of tourism, all the scenic spots can be eliminated and the remaining 50 scenic spots can be processed by cluster analysis to get the coordinates replacing two or more polygons in Table 1.

Table 1. 51 spots after clustering data

\begin{tabular}{|l|l|l|}
\hline Five-point clustering $(29,30,32,33,34)$ & 26 & 25 \\
\hline Four-point clustering(23, 26, 27, 28) & 18 & 31 \\
\hline Four-point $(15,16,18,21)$ & 37 & 43 \\
\hline Three-point $(12,13,14)$ & 22 & 47 \\
\hline Three-point $(38,39,40)$ & 58 & 50 \\
\hline Three-point $(46,48,49)$ & 78 & 87 \\
\hline Two-point $(35,36)$ & 20 & 14 \\
\hline Two-point $(22,24)$ & 5 & 27 \\
\hline Two-point $(19,20)$ & 12 & 37 \\
\hline Two-point $(7,41)$ & 52 & 61 \\
\hline Two-point $(47,50)$ & 85 & 91 \\
\hline
\end{tabular}

\section{Results discussion}

After the clustering of the spots and the 18 spots that have not been found, the coordinates of the 30 new aerial tram stations are constructed. We can use the Matlab technique to process the data in 
Table 1. After clustering, the coordinates of the 30 SkyTrain stations are obtained and the Floyd algorithm is used to find the optimal path for the cableway construction. The distance is: $d=$ $445.0506 \mathrm{~mm}$, the actual distance $\mathrm{D}=44505.06 \mathrm{~m}$, the minimum required cost is $\mathrm{S}=4450506000$ Yuan.The result of the improved algorithm as shown in figure 1.

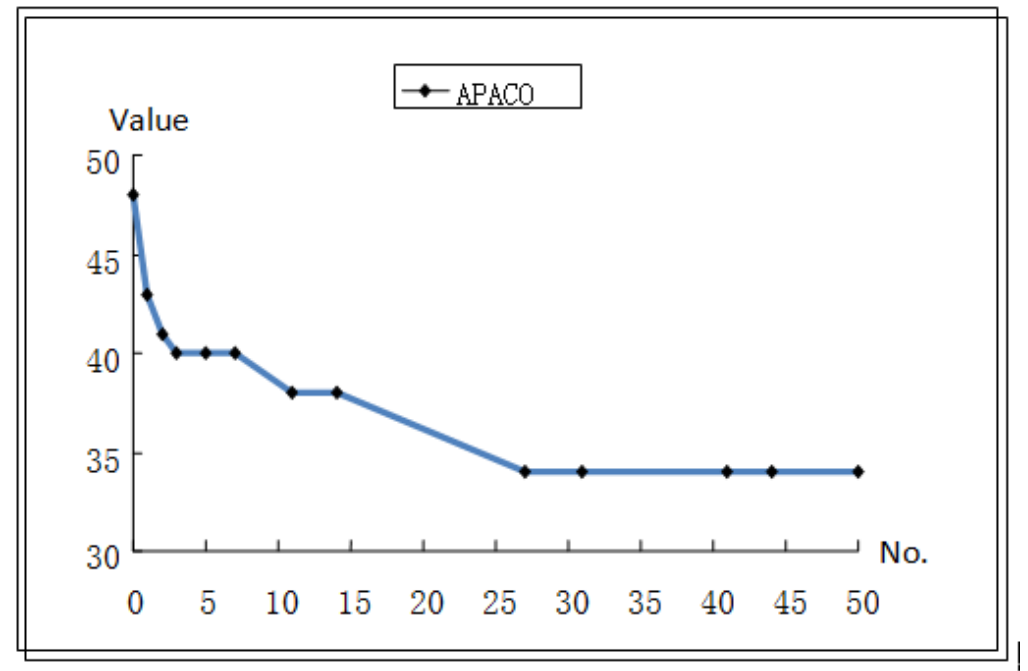

Figure 1. The result of The improved algorithm

\section{Conclusions}

Path planning technology is an important issue in the research of tourism path planning. The current research has mainly divided into two major categories: the global planning method and the local planning method. The global planning method is based on the geometry and topology method through the configuration space; and the local planning method are mainly based on the artificial potential field method based on the rectangular coordinate space. In this paper, the wisdom of rural tourism scenic spots laying ropeway abstract graph for the shortest path model, using the minimum spanning tree for expression. According to the needs of scenic area management department of Zhangjiajie, Floyd algorithm-cluster analysis method is used to establish and solve the model, and it can get the optimal solution of the problem. In view of the above advantages and disadvantages of the Floyd algorithm-cluster analysis model, this article gives specific improvements to make it more realistic and save the most money.

\section{Acknowledgements}

This work is supported by the Key projects for supporting outstanding young talents of universities in Anhui (gxyqZD2016574, gxyqZD2017138), 2016 Key projects of Humanities and Social Sciences in Anhui (SK2016A0905, SK2016A0910).

\section{References}

[1] Jeffrey S. Reid, James E. Kinney, Douglas L. Westphal, Brent N. Holben, Ellsworth J. Welton, \& Si-Chee Tsay, et al. (2003). Analysis of measurements of saharan dust by airborne and ground-based remote sensing methods during the puerto rico dust experiment (pride). Journal of Geophysical Research-Atmospheres, 108(D19), PRD 2-1.

[2] Stockford, C., Brandon, N., Irvine, J., Mays, T., Metcalfe, I., \& Book, D., et al. (2015). H2fc supergen: an overview of the hydrogen and fuel cell research across the uk. International Journal of Hydrogen Energy, 40(15), 5534-5543.

[3] Athanasios A. Pallis, Thomas K. Vitsounis, \& Peter W. De Langen. (2010). Port economics, policy and management: review of an emerging research field. Transport Reviews, 30(1), 115-161.

[4] Amin, S. M. (2011). Smart grid: overview, issues and opportunities. advances and challenges in sensing, modeling, 
simulation, optimization and control. European Journal of Control, 17(5-6), 547-567.

[5] Lessmann, C. (2011). Regional inequality and decentralization - an empirical analysis. Cesifo Working Paper, 44(6), 1363-1388.

[6] Magnussen, S. (2015). International Encyclopedia of the Social \& Behavioral Sciences, 2nd edition. International encyclopedia of the social sciences /. Macmillan Reference USA.

[7] Aydin, N. Y., Kentel, E., \& Duzgun, S. (2010). Gis-based environmental assessment of wind energy systems for spatial planning: a case study from western turkey. Renewable \& Sustainable Energy Reviews, 14(1), 364-373.

[8] Hashim, H., \& Ho, W. S. (2011). Renewable energy policies and initiatives for a sustainable energy future in malaysia. Renewable \& Sustainable Energy Reviews, 15(9), 4780-4787. 